\title{
A Study of Retrieval Methods of Multi-Dimensional Images in Different Domains
}

\author{
Shruti Garg \\ Dept of CSE \\ BIT, Mesra, Ranchi, INDIA
}

\begin{abstract}
Multiple amount of multi-dimensional images are designed and most of them are available on internet at free of cost. The 3D images include three characteristics namely width, height, and depth. The images which are created as 3D can describe the geometry in terms of $3 D$ co-ordinates. These coordinates help to obtain the object from the image much easier and accurate. In this paper, we presented a review about the Multi-dimensional image retrieval. Multi-dimensional image retrieval is a process of extracting the relevant $2 \mathrm{D}$ or $3 \mathrm{D}$ images from the huge database. To perform image retrieval process on large database, several methods like text based, Content based, Annotation based, semantic based, and sketch based were used. The image retrieval techniques are mostly used in the fields like Digital library, medical, forensic science, and so on. A systematic literature review has been shown for image retrieval methods reported on 2010 to 2017 . The aim of this article is to show the various concept and efforts of different authors on image retrieval technique.
\end{abstract}

Keywords-Image retrieval techniques; $3 D$ image retrieval; image retrieval survey

\section{INTRODUCTION}

The digital images are made by small and joined components is said to as pixels. These pixels are organized in horizontal and vertical manner, i.e. rows and columns of matrix. Every pixel includes three attributes $\mathrm{X}, \mathrm{Y}$ axis and gray value. The intensity of the radiation at the point of receptor exposure is corresponds to the gray value of every pixel. The channels Red, Green, and Blue are combined and forming the colored images and it also called as RGB images [1].

The process of initializing the image as input and getting an output as an attribute or the portion of an image is called Image Processing. The image processing techniques are used in the areas like image sharpening, color processing, pattern recognition, encoding, medical, and so on [2]. The image processing techniques are also used in high data processing and high-performance applications like face detection, face recognition etc. The algorithms used in the field of image processing can process the data of such application in high speed [3].

A technique with simple and global algorithm classes is image processing techniques. These techniques are much challenge and demanding on several systems like, smart devices, driver assistance systems, and medical imaging system. On smart devices like mobiles and tablets, there is need of efficient implantation for optimizing the battery. On the driver assistance systems, the process could be complete in the allocated period of time. The medical system contains huge amount of data, the image processing techniques need to process these data more efficient and faster. These image processing systems are not suitable for real time performance because these systems are mostly designed only to support desktop PCs. These limitations are fixed by inventing the FPGA chips and DSP processor which are based on computational platform [4] [5].

Various image data from different fields are collected and stored in the database, these image data are saved in database in the form of single dimension (1D) or multi-dimensional (2D or 3D). Latterly, these image data are much hard to access and reuse. The image retrieval techniques are enter in this situation and provide a solution for retrieving the stored images. Its main goal is to get an input and retrieve similar images based on the input [6]. On database there are different dimensional images are stored. From that, the single dimensional images only have one dimension called length. The 'line' is a good example for the single dimensional image, the dimension of line is length. The two-dimensional images have two dimensions namely length and width. The 2D images are mostly be in flat. The images which are derived with computer systems are mostly be in 2-dimension, because to build the 3-dimensional image it need to execute some complicated scripts. The 'square', 'triangle', and 'circle' are the good example for 2D images. The 3-dimensional images have three dimensions namely length, width, and depth [7] [8]. The 3-dimensional images take more memory for storing and high bandwidth for the transmission when comparing with 2dimensional images. The compression techniques are used in this situation for reducing the size of the 3-dimensional images. The 3D images are also said to as stereo image, because it includes two images of same object in a single frame. The compression process of $3 \mathrm{D}$ images is carried out based on the relationship between the left eye and right eye image [9]. The reconstruction of 3D image from $2 \mathrm{D}$ is also possible and it is already did by several researchers. During the $3 \mathrm{D}$ image reconstruction process, the dimensions of 2D image is extracted and then the depth is included with the extracted dimensions on the reconstructed image [10] [11].

These different dimensional images are extracted from the warehouse by using several image retrieval systems. Initially, while coding the image some of the information that is to represent the image are also coded. This information is used for matching the information to obtain the image and it is called retrieval system. The images and the information are 
coded with the imaging device called digital camera. The 2dimension and 3-dimension image samples are considered as features which are created by the capturing devices [12]. 3D image retrieval is an important problem in many applications, such as pattern recognition, image search engine, and some other applications [13].

Here in this article, a review about the various image retrieval methods for retrieving different dimensional images is presented. On the image retrieval process, the first step is to extract the features like geometric shapes, spatial and topological relationships, statistical measures, textures, and material colors of the image data. The information of multidimensional image will be created with these features. On the multi-dimensional feature space, the similarity among the input and feature model in the database will be defined. The results of retrieval process are generated by evaluating the similarity degrees [14].

The main goal of the multi-dimensional image retrieving techniques is to show the query or all available model in a useful manner. The retrieval system mainly focuses to describe the characteristics of object models. To achieve higher retrieval quality, the retrieval system should meet some requirements like robustness to linear transformations, invariance to noise, simplification and deformation and finally fast retrieval ability. The shape descriptor will provide better quality in terms of time and accuracy [15].

In market trading, the 3D trademarks are more significant and the registrations of 2D trademarks are also increasing significantly. The 3D trademark includes fixed length, width, height and the images are combined with shapes, text, graphics, symbols and colors. These significant data of 3D trademark are available at every angle [16]. The color attributes of image are one of the most common and basic features and it is used during the process of image retrieval and the spatial relationship among the pixels are defined by using the texture features of the image [17].

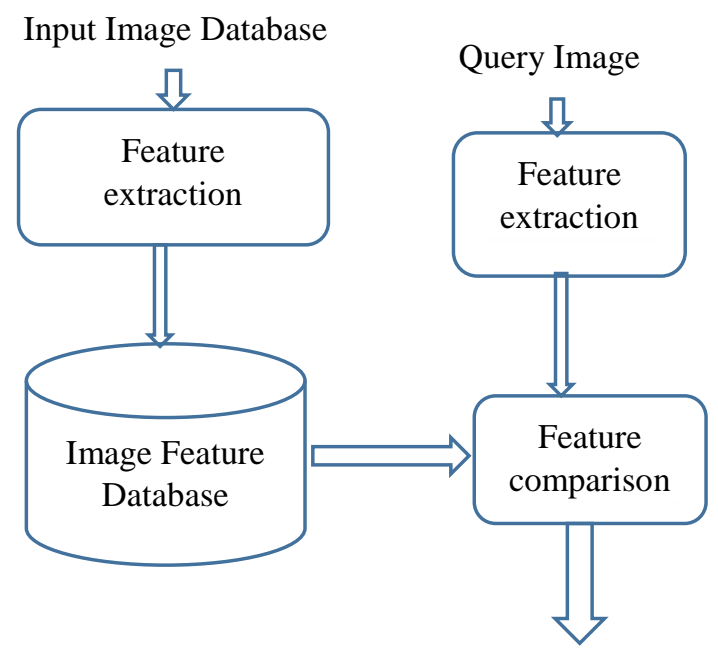

Output Image

Fig. 1. Basic image retrieval process
In the above fig 1 , the basic retrieval process of image is described. Initially on the first step, the text or an image is given as an input. Secondly, the features of the input text or image will be extracted. After extracting the feature from the input, it is matched with the feature of the images that are already stored in the database. At the final step, the feature of the images from the database which are matched with the feature of input is classified as the relevant image. These relevant images are given as the output for the users. Various retrieval methods are available for this retrieval process of image. Some of the most common retrieval techniques are described in this article on the below sessions.

The contribution of this review work is as follows: On section 2, we described some of the related works, section 3 describes the different image retrieval techniques, section 4 describes the some of the techniques used for retrieving the $3 \mathrm{D}$ images, and section 5 shows the metrics used for evaluating the performance of retrieval techniques.

\section{RELATED STUDIES}

In this session, some recent works based on image retrieval for retrieving various types of images is presented.

\section{A. Retrieval of Medical Image}

The amount of medical images are increasing enormously because of the high usage of medical imaging devices, with these devices hospital and other medical related systems producing huge amount of medical image each and every day. It is much hard to manage and analyze after which are stored in the database. These stored medical images can't be retrieve easily from large database, because its search space is much large [18]. For making efficient decision and diagnosis on medical field the authors of [19] presented an article for retrieving such medical images. In this article, the researchers used Fast Fourier Transform (FFT) for compress the convolution features into sequence of bits.

For the query medical image, they obtained the feature maps from the CNN which is already trained. These feature maps are obtained with the help of algorithm called optimal subset selection. With the binarization technique the layerwise global mean activations of feature maps are transform into binary codes. Here, the hash codes are achieved from feature vector and it is achieved without any training. They presented experiments of their method with two different image datasets (radiology and endoscopy). The result of the experiments shows that the framework proposed in this article performed well on features extraction and on hashing schemes. This hash code extraction framework is also compared with some other existing methods. The disadvantage of this framework is it can't work efficiently on very short codes, because of the use of Fourier spectrum to binary code transformation. On the future work of this article, the researchers planned to develop more feature vectors into hash code for extending the proposed framework and also planned to include some other frequency domain transformation methods.

Another article [20] presented a method to overcome the use of low-level hand-crafted features on medical image retrieval systems. The researchers of this article presented a 
method for retrieving the medical image large database called histogram of compressed scattering coefficients (HCSCs). The theoretical representation of medical image is obtained by performing the variation of deep convolutional networks and this process is said to as scattering transform. The attained scattering coefficients are compressed by performing the projection process and at last, from these compressed coefficients the bag-of-words (BoW) histogram is developed and it is taken as the features of medical image. The experiments of HSCSs is performed with several CT image datasets (EXACT09-CT, TCIA-CT, and NEMA-CT). The feature obtained using HSCSs performed well on some of the existing features like LBP, LMeP, LWP, and SS-3D-LTP. The future scope of this article is to derive much better projection technique to compress the scattering coefficients, derive better framework for compressed scattering coefficients for obtaining best features and improve the image representation by expanding the scattering transform.

\section{B. Retrieval of Remote Sensing Image}

The huge successful technologies like satellite and aerial vehicle producing uncountable quantity of remotely sensed images every day. There on Remote Sensing (RS) community it rises big issue while retrieving the RS image [21]. Some of the researchers focused on retrieving the remote sensing image is described as follows:

In article [22], a method is derived to retrieve the RSIR (Remote Sensing Image Retrieval) called two-stage reranking (TSR). Let assume that, on the initial retrieval we obtained knearest neighbors of a query remote sensing (RS), then the obtained neighbors are edited by the TSR using its editing scheme. The active learning algorithm is used to select the representations of RS images and based on the query image the clients will deliver binary labels of RS image. The selected $\mathrm{RS}$ images are used to train the binary classifier and its labels are used to classify the remaining neighbors. The neighbors for the exclusion will selected based on the results of classification and result of rank in the initial retrieval process. The next process of TSR is to reranking the remaining RS image (which are not excluded) and it is done by using multi similarity fusion reranking (MSFR). The TSR method uses the experience of users and relationships of images to perform the reranking process. Two kinds of RS images are taken by the researchers for showing the performance of TSR. They earned better performance when compared with other existing reranking approaches.

The scholars of [23] proposed a novel large-scale remote sensing image retrieval approach based on deep hashing neural networks (DHNNs). The DHNNs are derived by using two learning neural network namely feature learning and hashing learning neural network. The DHNNs is optimized in an end-to-end manner. With the supervision of labeled samples the feature extraction process and hashing mapping will be learned automatically instead of making the design of feature with high effort. The application field of DHNNs is extended by calculating it under some remote sensing cases namely scarce and sufficient samples. The DHNNs will be trained through transfer learning for the earlier case to makeup the labeled samples shortage. After this process, again the DHNNs is trained through supervised learning with the huge amount of labeled samples. The experimental process of the method proposed in this article is conducted with two public satellite image dataset. The experimental result shows that the DHNNs based method got better performance than other stateof the-art approaches. On the future work of this article, the researchers planned to discover more ways to train DHNNs by using labeled data with, minimal amount of errors, it makes the generation of data in low cost. Additionally, the authors planned to apply theirs DHNNs based system to more application with the base of remote sensing image by making it to support more applications.

\section{Retrieval of Crime Scene Image}

The growth of the technology is increasing day by day, at the same time the crime is also increasing. The identification process of criminals is a much complex task. During the investigation of crime, the images that are gathered from the crime spot is stored on huge database and it will be retrieved whenever the need of that images. The crime scene database includes some details like, photographs, images of the criminals or crime scenes collected during investigation by the investigators. The culprits are may find out by matching the current crime scene image with other previous crime scene image. The software with convenient technologically can also help the investigators to make right move on the investigation. The similar image retrieval of crime scene can help to reduce the time of the investigators [24].

The method presented in article [25] for classifying the crime scene images by efficiently describing the texture with DCT-based texture feature. The researchers of this article designed an algorithm based on texture feature for describing the crime scene images by extracting the features of images. Initially, on the description of crime scene images the GIST descriptor is utilized and the color histogram is also included. It helps to describe the crime scene image in various view like color, texture, and structure of scene. For classifying and retrieving the crime scene images, the SVM classifier is used. The combined feature algorithm proposed in this article performed well on retrieving the crime scene image with above $15 \%$ of increment on retrieval process while comparing with single-feature-based algorithm. There also obtained 3\% of improvement on precision by using SVM classifier.

On this analysis it is that found another notable article [26] for retrieving the shoe print image of crime scene. In this paper, for improving the performance of retrieval process the researchers used hybrid features of region and appearance of image on their manifold ranking based method. The manifold ranking based method proposed by the authors will perform retrieval process on shoe print images with high performance. The authors estimated the matches among the hybrid features and local features of shoeprints present in the crime image. But there have semantic gaps between the low-level and highlevel concepts. For improving performance, it needs to focus on sematic gaps between the feature concepts. So, the manifold hypothesis is applied on low level feature and semantic high-level space is applied on the proposed highlevel feature method. On the ranking function there some other classification of prior knowledge is included by the researchers. First one is, high score for the images in the database which are much closer to the feature of query image. 
Second one is, based on the opinion of the forensic expert the shoeprints are ranked if there are lot of foot prints are present in a single crime scene because it needs to consistent with the expert's opinion. In this article, a score called opinion score is described. It indicates the score that is given for every shoeprint in the crime images by the forensic experts. By ensuing above all constraints, the image which contains related shoeprints can be identified and ranked. At result, it provides the better similar images. They conducted experiments with real crime dataset and their algorithm got better performance of above $93 \%$. On their upcoming work, they planned to minimize the computation cost and maximize the performance by adaptively setting the parameters.

\section{Image Retrieval System on Cloud Environment}

Many smart devices are built with minimal amount of resources like, RAM, battery life, storage, computing ability, and so on. The smart device users may not have sufficient power to handle huge process like image processing, storing, and searching. So, the smart device users mostly use cloud computing technology for some works like, storing and searching. It performs with high performance on classical computing technologies and provide customization in cloud computing [27]. Here, for retrieving the image from large database cloud, various methods are used. Some of the image retrieval schemes that are recently proposed by various authors are explained below.

In [28] the authors proposed a scheme that supports CBIR over the encrypted images without revealing the sensitive information to the cloud server. In this article, the feature vectors are safeguarded with secure KNN algorithm. It helps to rank the images during the retrieval process without any communication problem by the cloud server. The localitysensitive hashing is used to build the pre-filter to separate the similar images. The method of this article includes two layers namely upper layer and lower layer. The pre-filter tables are located in the upper layer for enhancing the search efficiency. The lower layer contains one to one map index for ranking the search results. For examine the efficiency, here two typical visual descriptors are defined based on MPEG-7. The authors of this article telling that their method is simplified to the Euclidian distance based CBIR methods. On their future work, they planned to enhance the security level of extracted features from the images and also additionally they planned to make their system to extract features from encrypted images.

The researchers of article [29] construct a novel joint semantic-visual space by leveraging visual descriptors and semantic attributes. It merge the indexing and the attribute as a framework for restricting the semantic gap. This framework tends to improve the flexibility of Coherent Semantic visual Indexing (CSI), it increases the accuracy by boosting the retrieval process with the help of binary codes. Some of the contributions are added in the proposed method of this article. Firstly, joint semantic and visual descriptor space are detected by using the interactive optimization. Secondly, they demonstrate the union of their optimization algorithm by describing the solutions provided by their method for several iterations. Thirdly, they merged two systems namely spectral hashing and joint space system. At last, they build a cloud system for providing the online multimedia services. The best performance of their methodology is shown on their experiments. They conducted experiments with publicly available datasets and they proved proposed method is better than the existing methods. The researchers of this article say that their cloud-based image retrieval system better for using traditional server and efficient for work with high loads on cloud image retrieval.

\section{ClassifICATION OF IMAGE RETRIEVAL TECHNIQUES}

The image retrieval techniques are designed to search, browse, and retrieve relevant images from large databases. In this session, we shown that some of the popular image retrieval techniques that are used for processing the retrieval task. Some of the techniques which are covered in this review is described below,

- Text based image retrieval

- Annotation based image retrieval

- Semantic based image retrieval

- Sketch based image retrieval

- Content based image retrieval

Every technique has unique procedure to handle the retrieval process. Which will be explained on later sessions, and here on below figure 2 , different techniques and its features are described in a diagrammatic form.

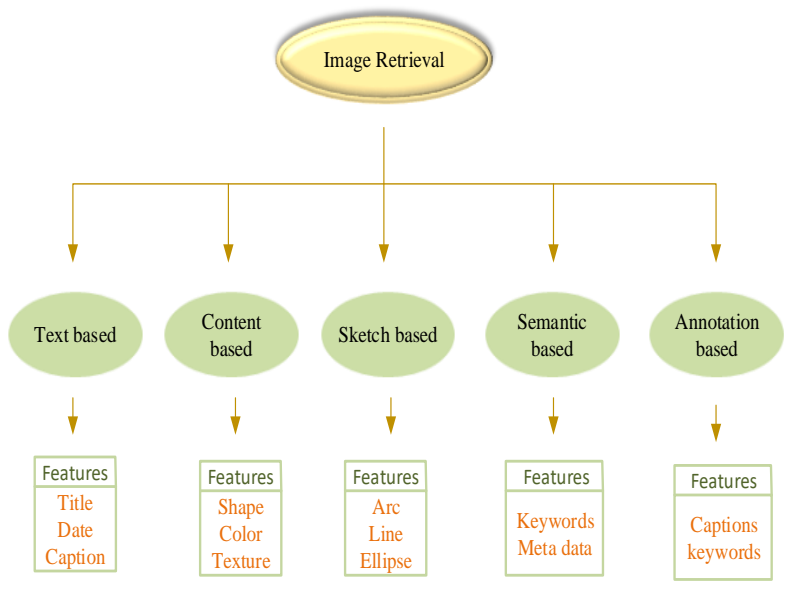

Fig. 2. Different classification of image retrieval techniques and its features

\section{A. Text based Image Retrieval}

This text-based image retrieval (TBIR) methods shown in figure 3 are mostly used in the year of 70's, it retrieves the image based on the feature of image like title, creation date, modification, deletion, and caption. This technique is also said to as description-based image retrieval. XML documents with images and having particular multimedia query can also retrieved by using the text-based retrieval method [30] [31]. With the inputted text, the textual and visual content descriptors are made for retrieving the images. These descriptors are transformed to vector format and also computed and changed into vector representation for the images already saved in the database. When users search for the image, they inputted the query and the vector for the query 
is generated, then it is matched with the already stored vectors in the database. Two different set of images with various weights are obtain as the result on text-based methods. The final image list is obtained by combining the two different set of images in the meaningful way [32]. The text-based methods work by combining the input text query and the descriptions of images. This technique only accepts text-based queries for retrieving the images. Some sample text-based queries are as follows, "search results for animals", "search results for animals added on 2018-05-25".

Main problems of the query by text:

- Unexpressed feelings, emotions

- Many ways of saying the same thing

- Synonyms and homonyms

- Misspellings

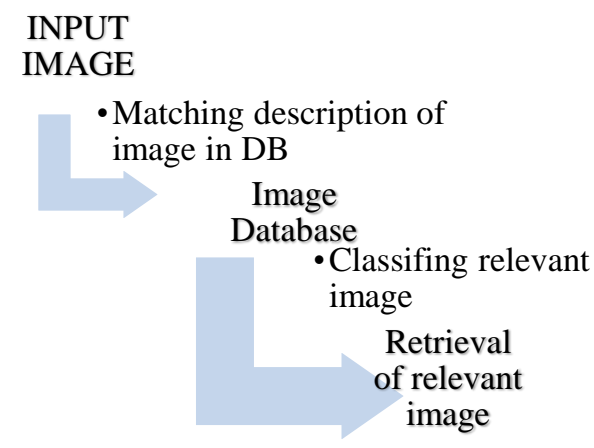

Fig. 3. Basic retrieval process of text-based image retrieval

\section{B. Content based Image Retrieval}

The content-based image retrieval systems are different than from text-based retrieval system because content-based system get the image as input and retrieve the similar images based on color, texture or shape shown in figure 4. Input of this type retrieval systems is look like "search for the image which containing animals" (i.e. It will be an animal image). It provides the result as various animal image similar to the input [33]. In this method, the retrieval process is carried out based on the features of the images, it uses the visual contents of the input image as the feature. For extracting the features of the image, feature extraction module is used, it extracts features from the set of images [31].

\section{1) Color}

CBIR retrieve the images based on three features of image, among one is color. It is a low-level feature, which is mainly used in the retrieval process because it is invariant to size and orientation. The color histogram of the images will be calculated and color values will be obtained. The similar color values among the input image and images stored in the database are classified. The color proportion of images be also calculated with the region and relationship between several color regions.

\section{2) Texture:}

Texture is another common feature of image, it indicated the objects present in the image and the correlation among the nearby environment. The object present in images can easily be identified with the texture. Still there doesn't have any universal definition for texture feature. It includes several basic primitives and it describes the object's structural arrangements and the correlations. The visual patterns of the images will be easily identified with the textures. The texture of the images is defined by text and it is stored in the sets based on the number of textures detected in an image. These sets help to define the texture and locate the texture of images. There also have some methods used on early days for detecting the texture of image, some of them are Cooccurrence matrix, Laws texture energy, and Wavelet transform.

\section{3) Shape :}

The shape feature indicates the shape of the objects, it will provide some useful information for retrieving the image, because with the shape of an object the humans can easily identify the objects without any other information. It is much differ from already revised features color and texture, shape additionally describes the semantic information. The shape features are classified into two types, boundary based, and region based. The shape doesn't indicate the shape of an image, it indicates the particular location of objects present in the image. By applying the segmentation or edge detection on an image the shapes will be determined. Other shape-based image retrieval systems identify the shapes by using the shape filters. The obtained shapes must be invariant to translation, rotation, and scaling [34] [35].
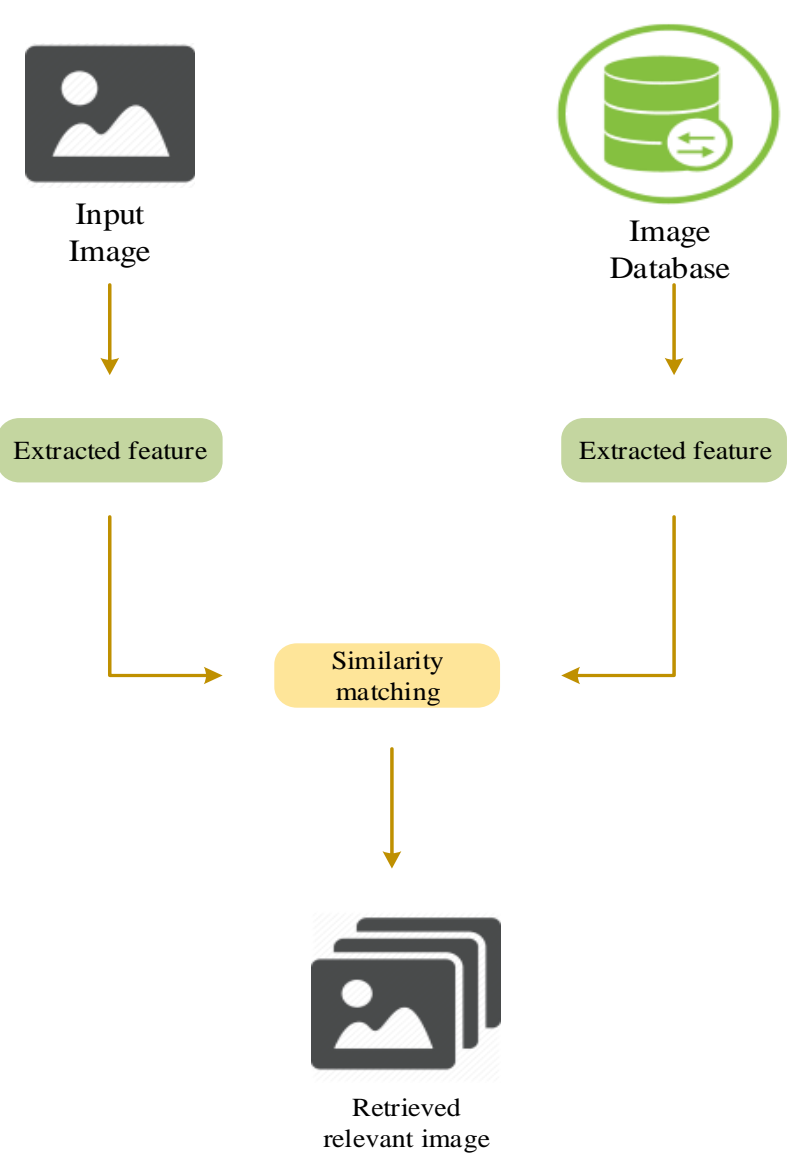

Fig. 4. Basic retrieval process of content-based image retrieval 


\section{a) Different Approaches on Content-Based Image}

Retrieval

Various authors are already focused and designed several content-based techniques, some of them are explained as follows. Munish Kumar et al [36] designed a content-based image retrieval system which uses two methods for extracting the features namely, SIFT (Scale Invariant Feature Transform) and ORB (Oriented Fast Rotated and BRIEF). Here, they used SIFT and ORB for detecting and describing the feature of images. The method ORB utilizes two features of image called FAST and BRIEF. They also used clustering algorithm Kmeans for analyzing the data and to generate several clusters with the help of descriptor vector. For enhancing the performance of their system, they minimized the length of the feature vector by using the LLP (Locality Preserving Projection). For classifying the similar images from the DB, they utilized several classifiers like BayesNet and KNN.

Atif Nazir et al [37] presented a new CBIR technique to fuse color and texture features. The authors of this article initially extracted the color information and texture features by using the $\mathrm{CH}$ (Color Histogram), transformation technique DWT (Discrete Wavelet Transform), and EDH (Edge Histogram Descriptor). The features which are derived from every image is saved on the DB as the feature vector. This paper exposed a method for retrieving image which works with the base of color and texture of image by using the combined local and global features. By combining the several features like color and text can provide better retrieval results and human visual system. The researchers of this paper, they extracted the local features by using the edge histogram and by using the transformation technique and color histogram the global features are extracted. The authors of this article used feature vector created with proper color and feature and relationships of images are equated by Manhattan distance.

Peizhong Liu et al [38] presents a method for CBIR system by integrating high- and low-level features from CNN and DDBTC (Convolutional Neural Network and DotDiffused Block Truncation Coding). The researchers of this article derived the low-level features like texture and color by using VQindexed histogram from DDBTC bitmap and some quantizers. The human perception can efficiently capture by using the features derived using CNN. They created deep learning two-layer codebook features by combining the features of DDBTC and CNN. The dimension of the feature is reduced for enhancing the retrieval rate. The strong feature is formed by GL-FCF and TLCF, with these strong features the overall retrieval rate will be increased. The method presented in this paper, discard some of the irrelevant images at the initial stage by using the hierarchical structure which they build on initial stage. The feature dimension is reduced by using DL-TLCF, it minimizes the comparing period. They initialize the normalized weights for creating the feature DLTLCF, by creating this feature the performance of their method will improve more significantly.

\section{Sketch based Image Retrieval}

This retrieval method is much differ from all other methods, it gets query image as sketch shown I figure 5. The sketch image contains the hand drawn objects, based on this sketch-based objects the images will be retrieved. The sketchbased image retrieval (SBIR) system is considered as a young research field, because of the usage and capable in various areas like searching and pattern detection [32].

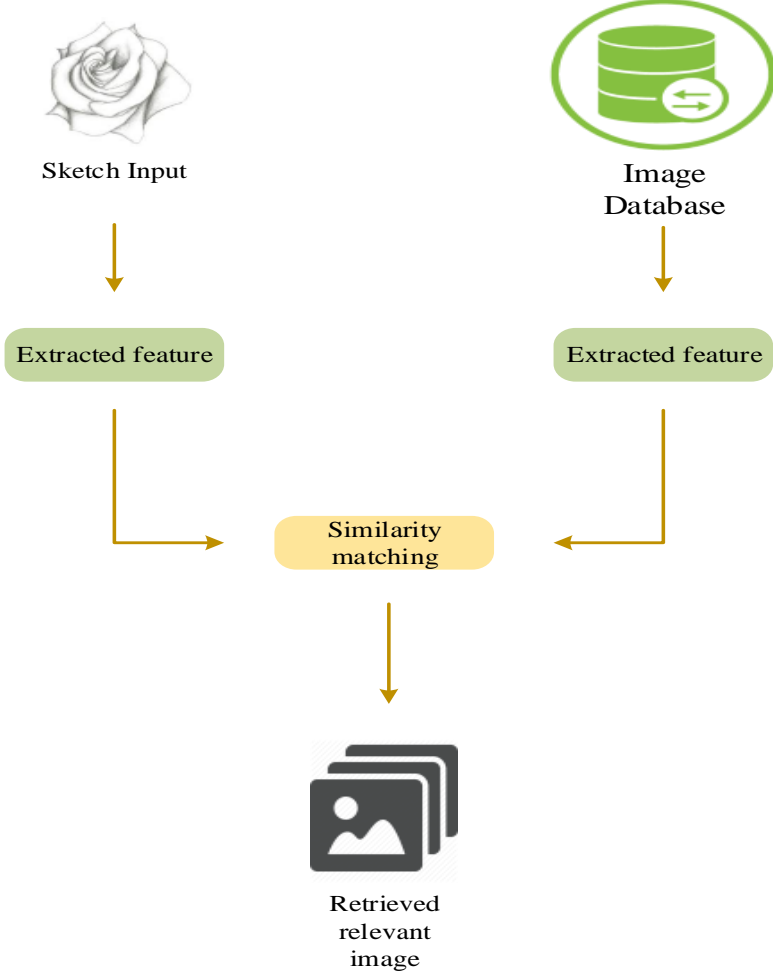

Fig. 5. Basic retrieval process of sketch-based image retrieval

On the previous method (CBIR), it utilizes image as a query. It is not easy for the users to get such picture which satisfies their need or the picture they are looking for. For overcome such issue the SBIR is introduced. It is much easier to draw a sketch based on the image that the user looking for. Most of the applications like CAD and 3D model retrieval methods utilize the sketch as the query for their process [39].

The sketch-based image can easily express and deliver the meaning and the intention of users. So, most of the multimedia contents retrieval systems are derived based on SBIR technique. In digital libraries, there is a huge collection of multimedia contents like image, video, and other data are stored by numerous users through the internet. So, sketch and text-based retrieval systems can perform excellently in digital libraries [40].

\section{a) Different Approaches on Sketch-Based Image} Retrieval

SBIR systems are widely used on different types of applications and especially on the situation of querying image not available. Because of the development of touch screen and smart phone technologies it is much simple for creating the sketch. Some of the research made by several authors on SBIR systems are described below.

Yonggang Qi et al [41] proposed a method for SBIR system based on the Siamese network. The main goal of this introduced system is to pull the output feature vector to nearby 
input sketch-image which are characterized as similar and take away from the input sketch if it is unrelated. These processes are carried out by two CNN which is connected with one loss function. The Siamese CNN in the proposed technique of this article can solve domain shift problems. The functions of this method will plot the input patterns into the target space, it helps to estimate the semantic distance in the input space. The main concept of this method is to pull the similar image much closer to input sketch and make away if the image is dissimilar. The researchers of this article telling that their method is introduced for overcome the issues of geometric distortions by absorbing the both positive and negative training pairs.

Jose M. Saavedra et al [39] proposed a novel local method based on detecting key shapes. This work focus on the fundamental information in terms of key shapes and local data in terms of local descriptors. The method proposed in this article can maximize the retrieval performance by describing the key shapes of the structures of object present in the image. It identifies some of the shapes that present in the sketch like line, arc, and ellipse.

The author of the previous [39] article Jose M. Saavedra presented another paper [42] for retrieving the image based on sketch. In this article, the authors exposed the improved version of Soft Histogram of Edge Logal Orientations (SHELO) for defining the sketches. They exposed their enhancements through two aspects. I.e. detecting the sketchbased representations with best technique and best normalization strategy of SHELO. To fulfill their first aspect, they introduced an approach called sketch token, it focused on identifying the contours of images in terms of mid-level features. To fulfill their second aspect, they improved the retrieval efficiency by establishing square root normalization.

Xueming Qian et al [43] presented a method based on ReRanking and Relevance Feedback. This method contains three several methods namely, image grouping, re-ranking via visual feature verification (RVFV), and contour-based relevance feedback (CBRF). The image grouping method aims to identify similar images for creating relevant feedback. The noisy images are eliminating by RVFV and also it makes the high rank images much applicable to the sketch query. The CBRF method identify similar image by using the contours of high ranked images which are already ranked by SBIR system. They are initiating the CBRF phase by eliminating the dissimilar images by applying RVFV. They work out their both system in offline and they are saying that the sketchbased methods will get back much preferred images.

\section{Semantic based Image Retrieval}

The semantic based image retrieval techniques work based on the keywords of images shown in figure 6. It gets input as image and keyword or only keyword. Most common search engines such as Google and Bing are using keyword-based search techniques for image retrieval process.

This type of image retrieval system works based on the keywords that are already stored by the users while creating the image. It matches the input keyword from the user with the keywords of images stored on the database and retrieve all images with similar keywords. But, this method is also having the possibilities for getting results with irrelevant images.

The [45] performance of semantic based image retrieval systems are better because of some reasons,

- While users searching for the images, if any mistake done on the spell of keyword it affects the retrieval system by retrieving dissimilar images.

- Sometimes it needs to specify the keywords for the images with natural language by the users, it may difficult to the users because the users need some knowledge about the natural language.

- The process of detecting the applicable keywords is much difficult for describing the image.

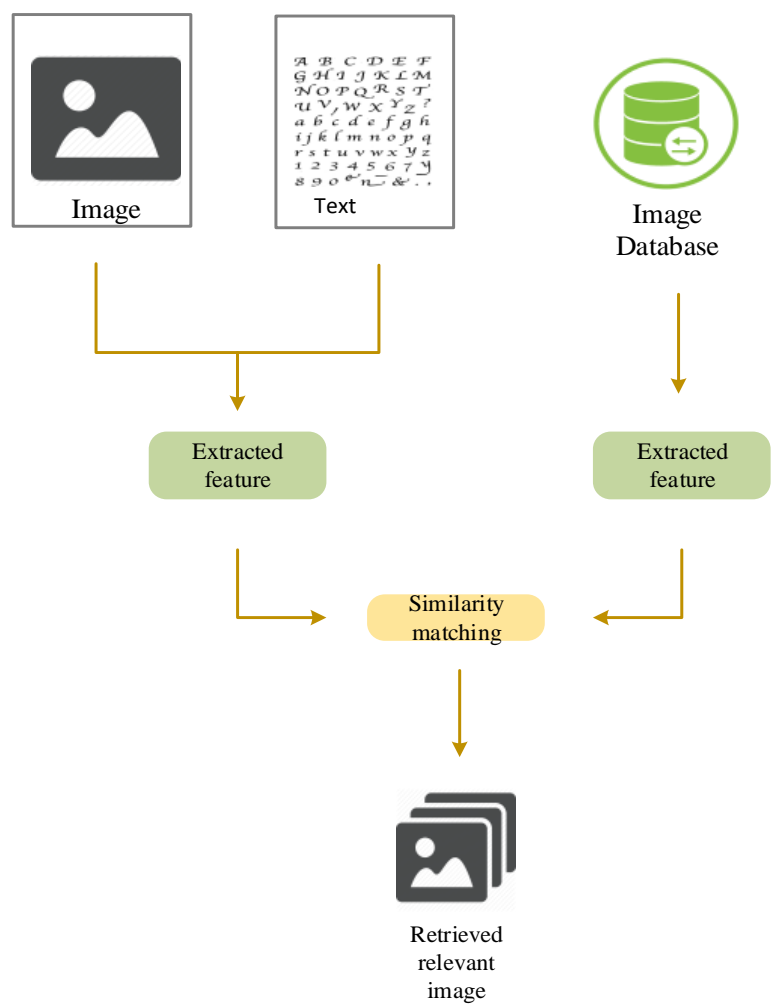

Fig. 6. Basic retrieval process of semantic based image retrieval Retrieval

a) Different Approaches on Semantic based Image

Because of the huge innovations of hand-held technology, inestimable quantity of images is available on web and it can also simply shared and uploaded by the users through the internet. There are several researches are directed on the field of image retrieval, but still efficient technique is not in sight. The [44] main reasons behind this deficiency is unavailability of literal information, nonspecific image data, different format of images, and low-level features of images. For solving such limitation semantic based retrieval techniques are introduced. Some of the research already conducted based on semantic based image retrieval techniques are explained below.

R.I.Minu et al [44] presented a semantic method for Ontology Based Image Retrieval System. One of the most 
investigated research field is image retrieval. In this article, the authors trained their system syntactically and semantically for designing a retrieval system with better accuracy. They focused on the retrieval of image on flower domain. They created the ontology for the flower domain and compared with its feature ontology. The researchers of this article telling that the created ontology can use with any type of retrieval process as a backend process. The method introduced in this article is much suitable for web-based image retrieval and they created the ontology in OWL language.

Umar Manzoor et al [45] proposed An Ontology based Approach which uses domain specific ontology for image retrieval relevant to the user query. This method accepts either text or an image as input. This method designed based on hybrid approach and it classify the similar images with the help of shape, color and texture. There is a matching module for matching the keywords/features, it gets the input from the query engine and it uses same process for retrieving the relevant images.

Anshy Singh et al [46] introduced a method called image crawling mechanism for retrieving the relevant images from web. The authors of this article focused on two major problems occurs while retrieving images from web, namely, freshness and redundancy. The authors also saying that their method can obtain text information present on the web. So, it can be used as an efficient image search engine. The mechanism of this article works based on the textual information available on nearby images on the web.

Min Wang et al [47] proposed a method for retrieving the remotely sensed images through matching the scene semantic information of the image. Initially, the low-level visual features in the image will be obtained with visual feature extraction technique and recorded on multilevel spatial semantics. This process includes some other methods like spatial relationship inference, and SS modeling. This scene semantic based retrieval works based on some features like object area, topology, and orientation of image. The authors saying, they implemented this method by using prototype system, it gives high accuracy rate on retrieval process. The method introduced in this article only supports to retrieve 10$30 \mathrm{~m}$ remote sensing images. It is mainly because of the matching based spatial resolutions and classification based on the object of image. By using this method for retrieving the remote sensing image will get high accuracy because of the usage of $30 \mathrm{~m}$ images.

\section{E. Annotation based Image Retrieval}

Generally, the users will give text as an input for textbased image retrieval systems for retrieving the images from the local DB or by browsing. This type of systems includes several problems on retrieving the images. Some of the problems are solved by annotation-based image retrieval system, it uses the metadata of images. The metadata includes presentational markup, comment, and explanation, these are attached while creating the image by the designer. The annotations may indicate the small piece of original information of image [48].
Annotation-based image retrieval (ABIR) basically uses text retrieval techniques on textual annotations of images that are included on the images by human [51]. The ABIR technique is new image retrieval method based on the annotation of image. The ABIR techniques includes two steps, automatic image annotation and query processing. The automatic process done by image segmentation and querybased process done by labeling process [52].

The ABIR will retrieve image through three ways,

\section{1) Free text annotation}

2) Annotation based on ontologies

3) Annotation using key words

Figure 7 shows the retrieval process in annotation-based method.

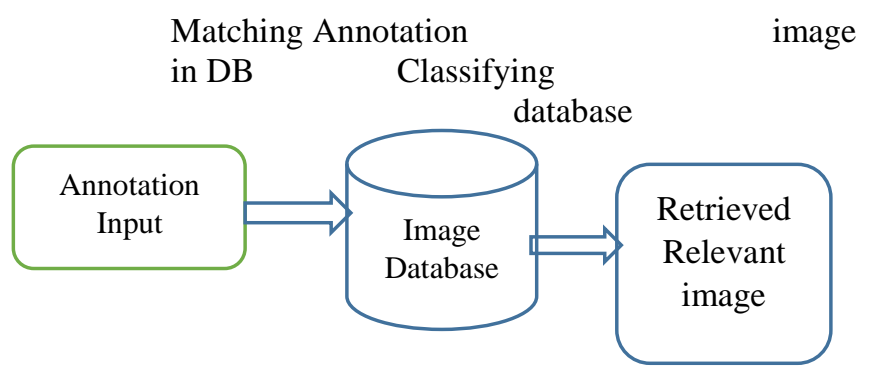

Caption/Keyword

Fig. 7. Basic retrieval process of annotation-based image retrieval

The free text annotation accepts any pattern of text or sentences as input. An ontology is a description of a conceptualization. The ontology includes entities, co-relations, and rules. On the keyword-based annotation, by using the keyword list that included in the image, every image will be annotated, and it is used as a keyword or annotation [32].

a) Different Approaches on Annotation-Based Image Retrieval

ABIR method based different approaches are described here. Hua Chen et al [49] presented an ABIR system based on $\mathrm{RDF}$ description. The researchers of this articles focused to provide a better concept for describing the semantics of image. On this image retrieval system, they included several levels for obtaining the annotation, i.e. concept, sentence, and instance. The annotation obtained through these levels are used as input/query for retrieving image and that annotations are stored in RDF triples. Some of the improvements that happen while using RDF description-based method are as follows,

- At the initial stage (concept and sentence), the semantic relations among the concepts are obtained, it refers some problems like synonyms and homonyms.

- At the instance stage, it calculates the amount objects like "how many people", "how many animals", and so on.

Himali P. Chaudhari et al [50] suggested an improved Markovian Model for ABIR using Multiple and Synonymous Keywords. This method is focused to improve the retrieval 
performance of state-of-art method (MSI). The method introduced in this article, initially identifies the keywords of user input and perform retrieval process and then show the retrieval result. The "recall" performance is reduced with the prevalence of synonyms. Same object can be described in several ways by the users. i.e., some users may have different knowledge, contexts, linguistic habits, and so on. So, such users will describe the things in different manner. This method expands the performance of MSI system by studying or obtaining several keywords from the input of users.

Deniz Kilinc et al [51] introduce an expansion and reranking approach for annotation-based image retrieval from Web pages. This approach works based on the text available on nearby image on the web and consider it as annotation. Such collected annotation includes useful information like copyright notice, date, and author. The selection approach presented by the researchers will enlarge the documents with WordNet and select the text and use it as annotation. This approach in applied on both document and input of users. By applying it on both sides can improve the retrieval performance than applying it on single side. Additionally, the selection approach provides maximum amount of terms per documents, it makes to look both the quires and documents are much better than the original.

Monica Hidajat [52] presented an ABIR method based on GMM and Spatial Related Object. In this approach, the image feature is clustered by using Gaussian Mixture Model (GMM) and for labelling methods such as, segmentation and multi class support vector machine is used as the classifier. The method introduced in this article includes two stage, 1) training and 2) testing and validation. On the first stage, the model is created for every keyword by using Support Vector Machine. On the second stage, the annotation and retrieval process are carried out.

\section{VARIOUS APPROACHES AND TECHNIQUES FOR RETRIEVING 3D IMAGE}

This was already explained in above text about the classification of various image retrieval techniques on previous session. Here, we discussed about some of the approaches and techniques that focused on $3 \mathrm{D}$ image retrieval.

The text-based image retrieval techniques are used in olden days and it is not focused by researchers because it has some limited features to retrieve the images. With the textbased retrieval techniques 3D images are can't be retrieve more efficiently. From this study it is found that some articles [53], [54] used content-based retrieval techniques for retrieving 3D images. Most of the researchers are working for retrieving $3 \mathrm{D}$ images using contend based method. At the same time, it is facing too much of challenges. The feature extraction process on $3 \mathrm{D}$ images are one of the most important challenge for the 3D image retrieval systems. The features extracting from the 3D images will be invariant of viewing angles, i.e. the objects need to visible for viewing from any angle.

Sketch based retrieving methods are also used for retrieving 3D images on [56], [57], [58]. In computer graphics, there is an important application is retrieving the $3 \mathrm{D}$ models from 2D. It is also important application on some areas like computer vision and information retrieval. By comparing the several methods, the sketch-based methods are better for the retrieval systems because of the sketch-based idea, sketch-based queries can easily be provided by users. The sketches are best for describing the shapes for retrieving the $3 \mathrm{D}$ images.

There are numerous tools are developed for creating 3D models and most of the $3 \mathrm{D}$ models are available on the internet at free of cost. Large set of 3D models are also available in market. For retrieving such 3D models is getting huge response and the demands of retrieval systems also increased. M. Elkhal et al [55] presented a method for retrieving the 3D images from large database using a method called level cut. This method works based on the binary image which is obtained from the $3 \mathrm{D}$ objects. It is achieved by connections in the set of the plans through the 3D object. A set of intermediate parallel plans created by the intersection through the 3D object a set of level cuts that utilized to indexing the 3D model.

Mostly for retrieving the 3D images the content based and sketch-based retrieval techniques are used. Because the 3D images are mostly retrieved based on the color, shape, and texture of the object present in the image. Text or keywordbased retrieval techniques retrieve based on the description or textual expression of images. The keywords of 3D images may not be described that the image is $3 \mathrm{D}$. So, it affects the efficiency of the 3D image retrieval system. I.e., it omits some $3 \mathrm{D}$ images during the retrieval process.

\section{VARIOUS PERFORMANCE METRICS USED BY IMAGE RETRIEVAL TECHNIQUES}

In this session, we illustrate the various performance metrics used by retrieval techniques for evaluating the performance.

True positive (TP): It indicates the state of right results obtained by the system.

True negative $(T N)$ : It indicates the state, if there is nothing to display as result and the system displays that no results.

False positive (FP): It indicate the state, if there is nothing to display as result and system displays some dissimilar results.

False negative (FN): It indicate the state, if there something is available, and system shows "there is nothing on result".

Precision: The ratio of obtained right results is described as the precision.

$$
\text { Precision }=\frac{T P}{T P+F P}
$$

Recall: The ratio of amount correctly obtained result among all right results. 


$$
\operatorname{Re} c a l l=\frac{T P}{T P+F N}
$$

F-measure: It indicates the average of both precision and recall.

$$
F-\text { measure }=\frac{2 *(\text { recall } * \text { precision })}{\text { recall }+ \text { precision }}
$$

MAP: Mean average precision is used to find the mean of the average precisions over a set of queries.

\section{CONCLUSION}

Nowadays, the usage of image retrieval techniques is increased because of the high demands of multimedia applications. In this research article, discussion has been done on different types of techniques mostly used in the field of image retrieval namely, text-based, content-based, sketchbased, semantic based, and annotation-based. Every technique has its own advantages and disadvantages. But the basic process of every techniques is same. i.e. these techniques initially extract the features from the input and then match it with the features of images present in the huge database and displays the matched images as result. These techniques can't satisfy the complete requirements of every uses. The requirements of the users will be increase day by day. So, different innovations are highly needed for satisfying the user requirements.

\section{REFERENCES}

[1] de Morais, Camilo de Lelis Medeiros, and Kássio Michell Gomes de Lima. "A colorimetric microwell method using a desktop scanner for biochemical assays." Talanta 126 (2014): 145-150.

[2] Das, Ananya, Abhishek Sahi, and Usha Nandini. "SAR image segmentation for land cover change detection." In Green Engineering and Technologies (IC-GET), 2016 Online International Conference on, pp. 1-6. IEEE, 2016.

[3] Rao, MV Ganeswara, Rajesh K. Panakala, and A. Mallikarjuna Prasad. "Image Processing using FPGAs: A Framework." SCESM-2016, at HIERANK Business School, Noida on (2016): 09-10.

[4] Rao, MV Ganeswara, P. Rajesh Kumar, and A. Mallikarjuna Prasad. "Implementation of real time image processing system with FPGA and DSP." In Microelectronics, Computing and Communications (MicroCom), 2016 International Conference on, pp. 1-4. IEEE, 2016.

[5] Membarth, Richard, Oliver Reiche, Frank Hannig, Jürgen Teich, Mario Körner, and Wieland Eckert. "Hipa cc: A domain-specific language and compiler for image processing." IEEE Transactions on Parallel and Distributed Systems 27, no. 1 (2016): 210-224.

[6] Foncubierta-Rodríguez, Antonio, Henning Müller, and Adrien Depeursinge. "Retrieval of high-dimensional visual data: current state, trends and challenges ahead." Multimedia tools and applications 69, no. 2 (2014): 539-567.

[7] Sefidmazgi, Nima Roohi, and Hussain U. Bahia. "Effect of compaction conditions on aggregate packing using 2-dimensional image analysis and the relation to performance of HMA." Materials and structures 47, no. 8 (2014): 1313-1324.

[8] Egenhofer, Max J. "Qualitative spatial-relation reasoning for design." In studying visual and spatial reasoning for design creativity, pp. 153175. Springer, Dordrecht, 2015.

[9] Lin, Yu-Hsun, and Ja-Ling Wu. "Quality assessment of stereoscopic 3D image compression by binocular integration behaviors." IEEE transactions on Image Processing 23, no. 4 (2014): 1527-1542.
[10] Willcocks, Chris G., Philip TG Jackson, Carl J. Nelson, and Boguslaw Obara. "Extracting 3D parametric curves from 2D images of helical objects." IEEE transactions on pattern analysis and machine intelligence 39, no. 9 (2017): 1757-1769.

[11] Tulsiani, Shubham, Abhishek Kar, Joao Carreira, and Jitendra Malik. "Learning category-specific deformable 3d models for object reconstruction." IEEE transactions on pattern analysis and machine intelligence 39, no. 4 (2017): 719-731.

[12] Jamkhandikar, Dayanand, Surendra Pal Singh, and V. D. Mytri. "Novel Method for 3D Objects Retrieval." International Journal of Computer Applications 128, no. 14 (2015).

[13] Guo, Kehua, and Guihua Duan. "3D image retrieval based on differential geometry and co-occurrence matrix." Neural Computing and Applications 24, no. 3-4 (2014): 715-721.

[14] Kao, Chi-Chou. "Shape-based 3D model retrieval system." International Journal of Computers and Applications (2016): 1-10.

[15] Alizadeh, Fattah, Alistair Sutherland, and Khaled Moradi. "Patch-Wise Charge Distribution Density for 3D Model Retrieval." International Journal of Modeling and Optimization4, no. 3 (2014): 233.

[16] Lee, Chu-Hui, and Liang-Hsiu Lai. "Retrieval of 3D Trademark Based on Discrete Fourier Transform." In International Conference on Mobile and Wireless Technology, pp. 620-627. Springer, Singapore, 2017.

[17] Yang, Jiachen, Bin Jiang, Baihua Li, Kun Tian, and Zhihan Lv. "A fast image retrieval method designed for network big data." IEEE Transactions on Industrial Informatics 13, no. 5 (2017): 2350-2359.

[18] Amin Khatami, Morteza Babaie, H. R. Tizhoosh, Abbas Khosravi, Thanh Nguyen, and Saeid Nahavandi. "A sequential search-space shrinking using CNN transfer learning and a Radon projection pool for medical image retrieval." Expert Systems with Applications 100 (2018): 224-233.

[19] Jamil Ahmad, Khan Muhammad, and Sung Wook Baik. "Medical Image Retrieval with Compact Binary Codes Generated in Frequency Domain Using Highly Reactive Convolutional Features." Journal of medical systems 42, no. 2 (2018): 24.

[20] Rushi Lan, and Yicong Zhou. "Medical image retrieval via histogram of compressed scattering coefficients." IEEE journal of biomedical and health informatics 21, no. 5 (2017): 1338-1346.

[21] Peng Li, and Peng Ren. "Partial randomness hashing for large-scale remote sensing image retrieval." IEEE Geoscience and Remote Sensing Letters 14, no. 3 (2017): 464-468.

[22] Xu Tang, Licheng Jiao, William J. Emery, Fang Liu, and Dan Zhang. "Two-stage reranking for remote sensing image retrieval." IEEE Transactions on Geoscience and Remote Sensing 55, no. 10 (2017): 5798-5817.

[23] Yansheng Li, Yongjun Zhang, Xin Huang, Hu Zhu, and Jiayi Ma. "Large-scale remote sensing image retrieval by deep hashing neural networks." IEEE Transactions on Geoscience and Remote Sensing 56, no. 2 (2018): 950-965.

[24] K. V. Shriram, P. L. K. Priyadarsini, and A. Baskar. "An intelligent system of content-based image retrieval for crime investigation." International Journal of Advanced Intelligence Paradigms 7, no. 3-4 (2015): 264-279.

[25] Ying Liu, Fuping Wang, Dan Hu, and Jiulun Fan. "Multi-feature fusion with SVM classification for crime scene investigation image retrieval." In Signal and Image Processing (ICSIP), 2017 IEEE 2nd International Conference on, pp. 160-165. IEEE, 2017.

[26] Xinnian Wang, Chi Zhang, Yanjun Wu, and Yingying Shu. "A manifold ranking based method using hybrid features for crime scene shoeprint retrieval." Multimedia Tools and Applications76, no. 20 (2017): 2162921649.

[27] Yuan Wang, Meixia Miao, Jian Shen, and Jianfeng Wang. "Towards efficient privacy-preserving encrypted image search in cloud computing." Soft Computing (2017): 1-12.

[28] Zhihua Xia, Neal N. Xiong, Athanasios V. Vasilakos, and Xingming Sun. "EPCBIR: An efficient and privacy-preserving content-based image retrieval scheme in cloud computing." Information Sciences 387 (2017): 195-204. 
[29] Richang Hong, Lei Li, Junjie Cai, Dapeng Tao, Meng Wang, and Qi Tian. "Coherent semantic-visual indexing for large-scale image retrieval in the cloud." IEEE Transactions on Image Processing 26, no. 9 (2017): 4128-4138.

[30] Tirilly, Pierre, Kun Lu, Xiangming Mu, Tian Zhao, and Yu Cao. "On modality classification and its use in text-based image retrieval in medical databases." In Content-Based Multimedia Indexing (CBMI), 2011 9th International Workshop on, pp. 109-114. IEEE, 2011.

[31] K. Shubhankar Reddy, and K. Sreedhar. "Image Retrieval Techniques: A Survey."

[32] Marshall, A. Malcom, and S. Gunasekaran. "A Survey on Image Retrieval Methods."

[33] da Silva Júnior, João Augusto, Rodiney Elias Marçal, and Marcos Aurélio Batista. "Image Retrieval: Importance and Applications." In Workshop de Vis ao Computacional-WVC. 2014.

[34] Naidu, M. Ekambaram, and Ravi Kumar Chandu. "A Complementary Review on Various Content Based Image Retrieval System Techniques." (2017).

[35] Wang, Xiang-Yang, Yong-Jian Yu, and Hong-Ying Yang. "An effective image retrieval scheme using color, texture and shape features." Computer Standards \& Interfaces 33, no. 1 (2011): 59-68.

[36] Kumar, Munish, Payal Chhabra, and Naresh Kumar Garg. "An efficient content based image retrieval system using BayesNet and $\mathrm{K}$ NN." Multimedia Tools and Applications (2018): 1-14.

[37] Nazir, Atif, Rehan Ashraf, Talha Hamdani, and Nouman Ali. "Content based image retrieval system by using HSV color histogram, discrete wavelet transform and edge histogram descriptor." In Computing, Mathematics and Engineering Technologies (iCoMET), 2018 International Conference on, pp. 1-6. IEEE, 2018.

[38] Liu, Peizhong, Jing-Ming Guo, Chi-Yi Wu, and Danlin Cai. "Fusion of Deep Learning and Compressed Domain Features for Content-Based Image Retrieval." IEEE Transactions on Image Processing 26, no. 12 (2017): 5706-5717.

[39] Saavedra, Jose M., and Benjamin Bustos. "Sketch-based image retrieval using keyshapes." Multimedia Tools and Applications 73, no. 3 (2014): 2033-2062.

[40] Song, Jinjoo, Hyeyoun Cho, and Sang Min Yoon. "Sketch-Based Shadow Image Retrieval for Digital Library." In Culture and Computing (Culture and Computing), 2017 International Conference on, pp. 127128. IEEE, 2017.

[41] Qi, Yonggang, Yi-Zhe Song, Honggang Zhang, and Jun Liu. "Sketchbased image retrieval via siamese convolutional neural network." In Image Processing (ICIP), 2016 IEEE International Conference on, pp. 2460-2464. IEEE, 2016.

[42] Saavedra, Jose M. "RST-SHELO: sketch-based image retrieval using sketch tokens and square root normalization." Multimedia Tools and Applications 76, no. 1 (2017): 931-951.

[43] Qian, Xueming, Xianglong Tan, Yuting Zhang, Richang Hong, and Meng Wang. "Enhancing sketch-based image retrieval by re-ranking and relevance feedback." IEEE Transactions on Image Processing 25, no. 1 (2016): 195-208.

[44] Minu, R. I., and K. K. Thyagharajan. "Semantic image description for ontology based image retrieval system." International Journal of
Applied Engineering Research 9, no. 26 (2014): 9332-9335.

[45] Manzoor, Umar, Mohammed A. Balubaid, Bassam Zafar, Hafsa Umar, and M. Shoaib Khan. "Semantic image retrieval: An ontology based approach." International Journal of Advanced Research in Artificial Intelligence (IJARAI) 1, no. 4 (2015): 1-8.

[46] Singh, Anshy, Shashi Shekhar, and Anand Singh Jalal. "Semantic based image retrieval using multi-agent model by searching and filtering replicated web images." In Information and Communication Technologies (WICT), 2012 World Congress on, pp. 817-821. IEEE, 2012.

[47] Wang, Min, and Tengyi Song. "Remote sensing image retrieval by scene semantic matching." IEEE Transactions on Geoscience and Remote Sensing 51, no. 5 (2013): 2874-2886.

[48] Kumar, G. Nandha, and V. Saranya. "IRMA-Improvisation of image retrieval with Markov chain based on annotation." In Information Communication and Embedded Systems (ICICES), 2014 International Conference on, pp. 1-7. IEEE, 2014.

[49] Chen, Hua, Antoine Trouve, Kazuaki J. Murakami, and Akira Fukuda. "An intelligent annotation-based image retrieval system based on RDF descriptions." Computers \& Electrical Engineering 58 (2017): 537-550.

[50] Chaudhari, Himali P., and Dinesh D. Patil. "Improved Markovian Model for Annotation Based Image Retrieval using Multiple and Synonymous Keywords." (2014).

[51] Kılınç, Deniz, and Adil Alpkocak. "An expansion and reranking approach for annotation-based image retrieval from web." Expert Systems with Applications 38, no. 10 (2011): 13121-13127.

[52] Hidajat, Monica. "Annotation Based Image Retrieval using GMM and Spatial Related Object Approaches." International Journal of Control and Automation 8, no. 8 (2015): 399-408.

[53] Qian, Yu, Xiaohong Gao, Martin Loomes, Richard Comley, Balbir Barn, Rui Hui, and Zenmin Tian. "Content-based retrieval of 3D medical images." In The Third International Conference on eHealth, Telemedicine, and Social Medicine (eTELEMED 2011), pp. 7-12. 2011.

[54] Gao, Xiaohong, Yu Qian, Rui Hui, Martin Loomes, Richard Comley, Balbir Barn, A. Chapman, and J. Rix. "Texture-based 3D image retrieval for medical applications." In IADIS Int. Conf. e-Health, pp. 101-108. 2010.

[55] Elkhal, Mustafa, Abdelghni Lakehal, and Khalid Satori. "A New Method For 3d Shape Indexing And Retrieval In Large Database By Using The Level Cut." Journal of Computer Science 10, no. 10, 2014.

[56] Wang, Fang, Le Kang, and Yi Li. "Sketch-based 3d shape retrieval using convolutional neural networks." In Computer Vision and Pattern Recognition (CVPR), 2015 IEEE Conference on, pp. 1875-1883. IEEE, 2015.

[57] Furuya, Takahiko, and Ryutarou Ohbuchi. "Visual saliency weighting and cross-domain manifold ranking for sketch-based image retrieval." In International Conference on Multimedia Modeling, pp. 37-49. Springer, Cham, 2014.

[58] Xu, Peng, Qiyue Yin, Yonggang Qi, Yi-Zhe Song, Zhanyu Ma, Liang Wang, and Jun Guo. "Instance-level coupled subspace learning for finegrained sketch-based image retrieval." In European Conference on Computer Vision, pp. 19-34. Springer, Cham, 2016. 